\title{
ESTUDO DO SISTEMA DE FILAS PARA REALIZAÇÃO DE EXAMES EM UM LABORATÓRIO EM PARAUAPEBAS-PA
}

\author{
Thaynara Lopes dos Santos (Universidade Federal Rural da Amazônia) sthaynaralopes@gmail.com \\ Cristine Sampaio Rocha (Universidade Federal Rural da Amazônia) sampaiorochac@gmail.com \\ Ellen Laena de Sousa Sousa (Universidade Federal Rural da Amazônia) ellenlaena@ gmail.com \\ Lucas Rafael Castro de Sousa (Universidade Federal Rural da Amazônia) lucasraf.castro@gmail.com \\ Diego Moah Lobato Tavares(Universidade Federal Rural da Amazônia) moah6@hotmail.com
}

\section{Resumo}

Em diversas situações do cotidiano é comum perceber esperas que geram filas, algumas são rápidas, mas outras ocorrem além do planejado, sobretudo, quando se trata de atendimento em redes públicas como é o caso do laboratório municipal, localizado na cidade de Parauapebas no estado do Pará. Este local foi escolhido como objeto de estudo principalmente por ser o único laboratório público da cidade e claramente perceptível as longas filas devido a demanda alta por atendimento e baixa capacidade do sistema em absorvê-la em curto tempo. A Lei 13.460 de Junho de 2017 dispõe dentre os direitos básicos dos usuários aos serviços públicos que os mesmos tenham previsão do tempo de espera para atendimento. Portando este trabalho foi desenvolvido aplicando os conceitos de teoria de filas com o propósito realizar uma análise do sistema de atendimento e propor uma solução para minimizar a espera dos pacientes ao realizar exames.

Palavras chave: (Teoria das filas; Filas em hospitais; Atendimento)

\section{Introdução}

Todas as pessoas já precisaram enfrentar filas em algum momento, algumas as enfrentam diariamente e até várias vezes no mesmo dia, seja em um banco, em supermercados, no trânsito ou em muitas outras situações que fazem parte do dia a dia.

Entre essas muitas situações nos deparamos constantemente com grandes esperas quando precisamos de atendimento médico, especialmente quando estes são realizados em hospitais pertencentes a rede pública de saúde. As esperas para a utilização dos serviços públicos formam grandes filas e como consequência estresse e em alguns casos até revolta por parte da população.

A teoria das filas pode ser definida como um método matemático que estuda todas as formas de espera, utilizando um modelo de filas para representar os mais diversos sistemas que detém filas do mesmo estilo que são utilizadas na prática. As fórmulas propõem modelos em que os 
sistemas devem funcionar considerando diversas circunstâncias (HILLIER e FREDERICK, 2006).

A saúde é um direito fundamental de todos os seres humanos e deve ser garantida pelo Estado de forma igualitária e universal com a aplicação de políticas econômicas e sociais. No Brasil esse direito é garantido através do SUS, Sistema Único de Saúde, que tem como um de seus objetivos oferecer assistências a população de modo a garantir a todos assistência integral a saúde (PLANALTO, 1990).

Na prática o sistema de saúde público no Brasil é bem diferente do que descrito na lei, não sendo tão estruturado como deveria, não dispõe de um sistema hierarquizado e eficiente, detém de uma grande necessidade em recursos e investimentos nos hospitais e até a falta de profissionais qualificados, são fatores que justificam a sua atual situação.

As filas geradas são inúmeras, seja para realização de exames, consultas ou na maioria dos casos para intervenções cirúrgicas, elas se dão quando um determinado número de pacientes precisa do mesmo serviço ou tratamento tendo uma demanda maior que a oferta podendo levar até anos para que alguns casos sejam atendidos.

A cidade de Parauapebas no Sudeste do Pará possui em média 208.273 mil habitantes levando em consideração ao último senso realizado em 2009 pelo IBGE, possui vinte e uma unidades de pronto atendimento de urgência (UPA's), uma unidade do sistema de atendimento móvel de urgência (SAMU) e um hospital geral, que tem à disposição apenas um laboratório municipal para atendimento das demandas geradas (DATASUS, 2019).

Considerada uma das grandes influências no estado devido a sua grande receita, o município possui uma estrutura precária no que diz respeito a atendimento no setor público de saúde gerando grandes transtornos à população que depende desse serviço, não correspondendo com a sua real situação econômica.

Com base no seu atual cenário e na grande espera gerada por filas em seus hospitais públicos para a realização de exames, foi aplicado um estudo que, através da Teoria das filas, busca analisar as filas geradas pela demanda para a realização de exames no laboratório municipal da cidade de Parauapebas, através da coleta de materiais no pronto socorro, propondo possíveis soluções para a melhoria na prestação do serviço.

Este artigo está estruturado em uma breve introdução do tema contendo o contexto, motivação e objetivo. A Seção 2 contém os alguns tópicos teóricos relevantes para o estudo, na Seção 3 
descreve-se metodologia utilizada. Na Seção 4 apresentam-se os resultados encontrados, e as propostas de melhoria. Por fim, a Seção 5 contém as considerações finais do estudo.

\section{Referencial Teórico}

\subsection{Teoria das filas}

Prado (2004) afirma que é comum deparar-se com problemas de fluxo e dimensionamento de soluções aparentemente complexas, ao realizar determinados estudos de planejamento. Sendo o cenário com diversas possibilidades, como fluxos de documentos em escritório, movimento de veículos, equipamentos e minério em uma mineração, dentre outros.

Esses estudos podem ser realizados para uma redefinição de layout, trocas de equipamentos, ampliações em uma fábrica, automatização, dimensionamento de uma nova fábrica, além de sua aplicação para otimização de serviços etc. Ou seja, ainda corroborando Prado (2004), ao ser definido um objetivo relacionado à produção ou qualidade de atendimento, o estudo visa definir a quantidade adequada de atendentes (equipamentos, veículos, pessoas etc.) a serem alocados no sistema de trabalho bem como o layout mais apropriado e o melhor fluxo. Aspirando que o sistema resulte um funcionamento eficiente. Um exemplo dos elementos de uma fila está representado na figura 01, a seguir.

Figura 01 - Elementos de uma fila

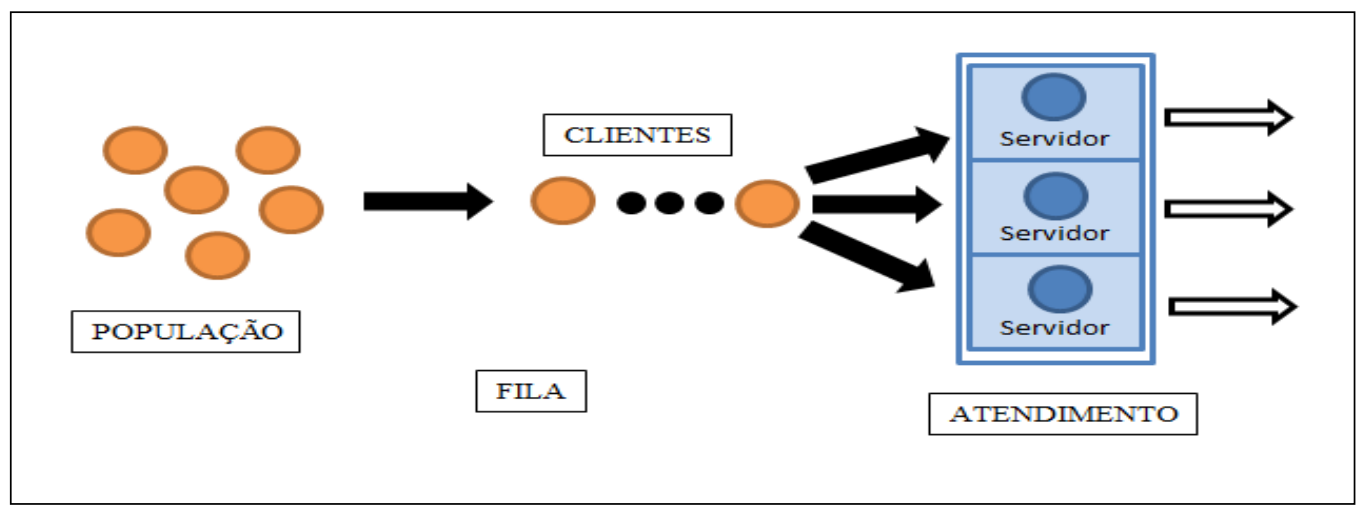

Fonte: Adaptado de Prado (2004)

\subsection{Características de uma fila}

\subsubsection{Clientes e Tamanho da População}

Prado (2004), afirma que o cliente é proveniente de uma população e está quando é muito grande é caracterizada como infinita. As chegadas dos clientes são independentes, uma vez que a chegada de um novo cliente não interfere no próximo. 


\subsubsection{Processo de chegada e Processo de Atendimento}

Para caracterizar corretamente um processo de chegada deve-se utilizar uma distribuição de frequência, como a distribuição normal, de Poisson, exponencial etc. Para quantificar esta variável é utilizada a letra grega $\lambda$ representando o ritmo médio de chegada e IC para o intervalo médio entre chegadas (PRADO, 2004). Podendo ser medido através do número médio de chegadas por dada unidade de tempo ( $\lambda$ - taxa média de chegada) ou pelo tempo médio entre chegadas sucessivas (IC intervalo médio de tempo entre chegadas) (GROSS et al., 2008; CAMELO et al., 2010).

A distribuição de Poisson, também pode descrever o processo de chegada, em variados modelos de fila, tendo relação com a distribuição exponencial. A exponencial faz o ajuste do tempo entre chegadas e a Poisson faz o ajuste do número de chegadas em um determinado intervalo de tempo. Bem como, no processo de atendimento pode-se utilizar a distribuição exponencial para indicar o tempo de atendimento do servidor em um modelo básico de filas.

\subsubsection{Número de Servidores e Disciplina da fila}

Segundo Taha (2008), a disciplina mais comum é "primeiro a chegar, primeiro a ser atendido", há também "último a chegar, primeiro a ser atendido", "serviço em ordem aleatória", "serviço por ordem de prioridade", podendo ser "preemptiva", onde o serviço em andamento é interrompido para atender quem acabou de chegar, como em prontos-socorros, ou "não-preemptiva", onde o serviço em andamento é concluído antes de atender quem acabou de chegar, entre outras.

No caso de fila com prioridade, conforme Arenales et al. (2007), há o caso com interrupção, no qual o usuário com maior prioridade é atendido assim que chega na fila, mesmo que um usuário de menor prioridade esteja sendo atendido, e no sistema sem interrupções o usuário de menor prioridade não pode ser interrompido até ser completado.

\subsection{Modelo $\mathrm{M} / \mathrm{M} / 1$}

Arenales et al. (2007), refere-se ao modelo de Fila M/M/1 como um sistema composto de apenas uma fila que é atendida por apenas um servidor, descrito por distribuição de Poisson de parâmetro $\lambda$, para processo de chegada, e o tempo de atendimento descrito por distribuição exponencial negativa, com parâmetro $\mu$. Não havendo limites para o tamanho da fila e nem para o tamanho da população de usuários.

Na tabela 01, a seguir, as equações que tratam as principais varáveis randômicas para população infinita (PRADO, 2004). 
Tabela 01 - Equações das principais variáveis randômicas de modelo M/M/1 com população infinita

\begin{tabular}{lc}
\hline Descrição & Equação \\
\hline Número médio de clientes na fila & $\mathrm{NF}=\frac{\lambda^{2}}{\mu\left(\mu_{-\lambda}\right)}$ \\
\hline Número médio de clientes no sistema & $\mathrm{NS}=\frac{\lambda}{\mu-\lambda}$ \\
\hline Tempo médio de espera no sistema & $\mathrm{TS}=\frac{1}{\mu-\lambda}$ \\
\hline Tempo médio de espera na fila & $\mathrm{TF}=\frac{\lambda}{\mu(\mu-\lambda)}$ \\
\hline Probabilidade de ter n clientes no & $\left(\frac{\lambda}{\mu}\right)^{n}\left(\frac{\mu-\lambda}{\mu}\right)$ \\
sistema & $\mathrm{Pn}=\frac{\lambda}{\mu}$ \\
\hline Taxa de utilização do servidor & \\
\hline
\end{tabular}

Fonte: Adaptado de Prado (2004)

\subsection{Modelo M/M/s}

Em sistemas de filas com mais de um servidor, Barbosa et al. (2009), cita Prado (1999), afirmando que os processos de chegadas e de atendimento se dão como no modelo $\mathrm{M} / \mathrm{M} / 1$, conforme distribuição de Poisson e Exponencial negativa, respectivamente, sendo a disciplina da fila do tipo FIFO e a taxa de utilização do servidor é dada por $\rho=\frac{\lambda}{s \mu}$, onde $s$ é o número de servidores. Quando se tem uma mesma taxa de utilização $(\rho)$, ao aumentar a quantidade de servidores, o tamanho da fila diminui e aumenta o número de clientes no sistema (PRADO, 2004).

Menores tempos de espera em filas e menores perdas de usuários no sistema, o que, consequentemente, exige maior investimento em capacidade do sistema, geram melhores níveis de serviço. Da mesma forma, os modelos com múltiplos servidores, tentam equilibrar o conflito entre os custos de fornecer um nível de serviço no sistema e os custos provocados pelos atrasos sofridos pelos usuários (ARENALES et al., 2007).

\subsection{Estudo das filas em Unidade de Saúde}


Mathias et al. (2015) afirma que a aplicação de modelos de teorias de filas na área da saúde tem sido um solucionador de problemas sobre o congestionamento e dimensionamento de demanda no sistema hospitalar.

Para Marinho (2004), a demora no atendimento exerce impactos significativos [...] com as perdas de eficiência geradas pelo eventual stress nos hospitais, pela desordem organizacional no SUS e pela pressão social decorrentes das filas. A perda de prestígio social do sistema de saúde também costuma ser decorrência de tempos de espera elevados e/ou imprevisíveis e/ou "injustos".

Apesar deste cenário Chin et al. (2014), afirma que estudos referentes a Teoria das Filas, com seu foco sobre a área da saúde, são escassos e muito recentes. Diante dessa realidade o presente estudo avalia a eficiência do sistema de atendimento hospitalar limitando ao processo de coleta para realização de exames laboratoriais.

\section{Metodologia}

\subsection{Escolha do local para o estudo}

Em conjunto com o professor orientador foram analisados os potenciais cenários para desenvolvimento do trabalho, destes um se destacou devido à ausência de previsibilidade das filas e um tempo de espera significativo pelos usuários do sistema, para coleta de materiais para exame do Laboratório Municipal, localizado no Hospital Geral de Parauapebas.

\subsection{Levantamento dos dados}

Ao escolher o local do estudo e obter a autorização do responsável do setor, foi possível colher os dados acerca do ritmo de chegada e tempo de atendimento. Em cada dia da observação um integrante da equipe se disponibilizou para medir e anotar o horário de chegada, início e fim de atendimento, para posteriormente calcular os respectivos ritmos. Os horários para a coleta dos dados foram definidos em consonância com a colaboradora responsável pelo setor, nos quais existissem um fluxo constante de chegada.

\subsection{Caracterização do sistema de filas}

Após a observação e coleta de dados o grupo de estudo realizou a representação simplificada do problema por meio de um modelo e posteriormente, considerando as disciplinas das filas (FIFO, LIFO, SIRO, PRI e GD), classificou o sistema adotado. Sendo que esta classificação poderia ocorrer de quatro formas. A saber: 
a) Fila única e um único servidor;

b) Fila única e múltiplos servidores em paralelo;

c) Múltiplas filas e múltiplos servidores em paralelo;

d) Fila única e múltiplos servidores em paralelo.

Foi utilizado o software Input Analyzer, ferramenta do software Arena® 11.0 versão Student, o qual fornece o resultado do teste Qui-Quadrado, o que permite analisar se o modelo estudado corresponde corretamente ao sistema. Para obter as medidas de efetividade de um sistema, foi utilizada uma macro configurada para tais cálculos, no software Excel.

\section{Estudo de Caso}

\subsection{Escolha do local para o estudo}

O laboratório Municipal de Parauapebas, localizado no Hospital Geral de Parauapebas é o único laboratório público na cidade. Nele são feitos exames de rotina e exames de urgência da maior parte da população do município.

Para os exames de urgência é feita a coleta de materiais em uma sala no prédio do pronto socorro do Hospital Municipal. Lá são coletados materiais como sangue, materiais fisiológicos, para exames solicitados em consultas no pronto socorro, 24 horas por dia. Esse material então é direcionado ao laboratório para serem realizados os exames, os quais geralmente são concluídos em uma média de duas horas.

A sala de coletas foi o local escolhido para a realização do estudo, visto que são casos de urgência nos quais os atendimentos são realizados em ordem de chegada, e apenas um servidor os faz, ademais, os pacientes que são atendidos no local já passaram algum tempo aguardando o procedimento para realização de consulta, por tanto, um atendimento rápido e de qualidade feito na sala de coletas é importante, principalmente, para evitar irritações advindas dos clientes.

\subsection{Levantamento dos dados}

A sala de coletas atende 24 horas, dividida em três turnos: matutino, vespertino e noturno, em cada turno um servidor realiza a coleta dos materiais necessários para a realização de exames laboratoriais. O layout da sala onde ocorrem as coletas está representado na figura 02. 


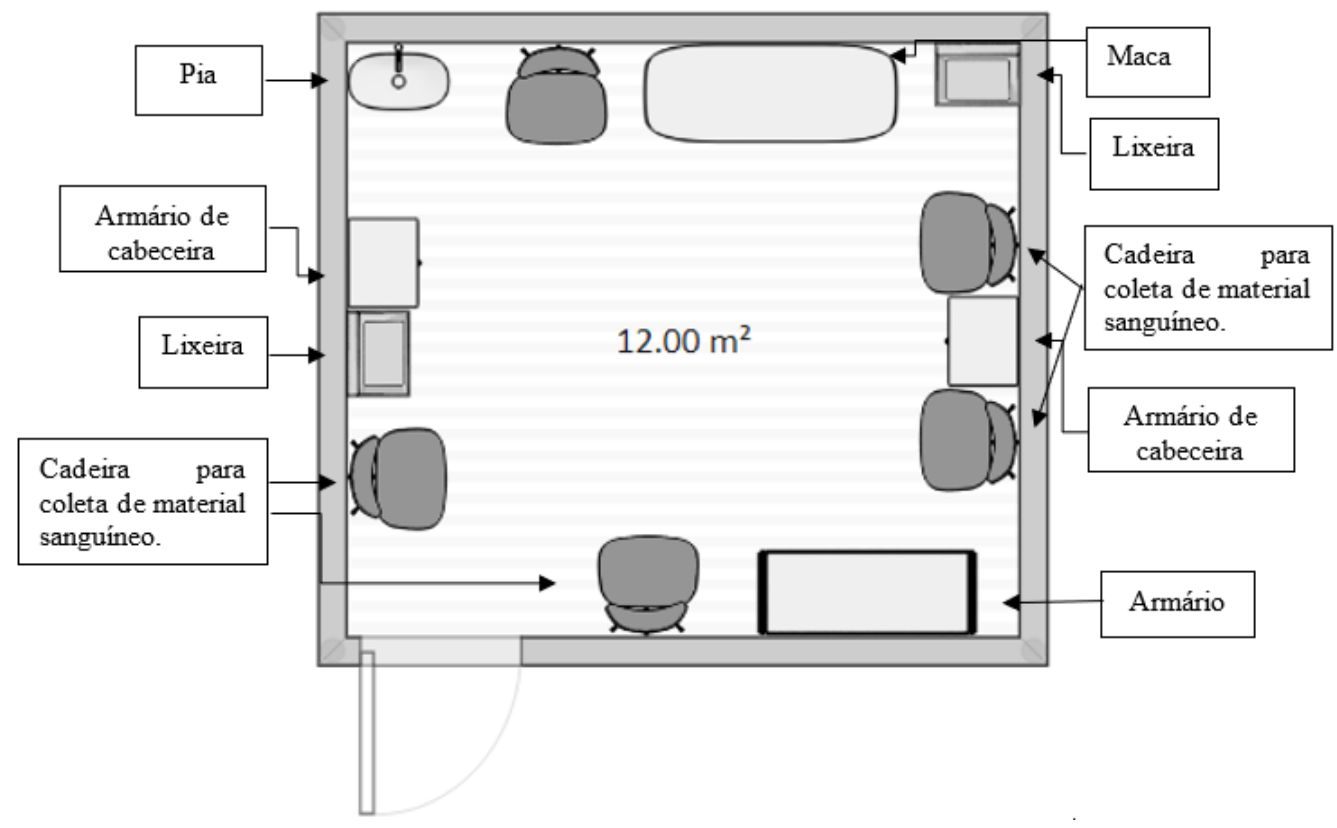

Fonte: Autores (2019)

Os dados foram coletados, no período da manhã, por meio da observação, utilizando o cronômetro para contabilizar os horários de chegada de cada paciente, bem como o horário de início e fim de cada atendimento. Foram coletados cerca de 300 dados, porém alguns horários precisaram ser descartados, devido à inconstância na chegada e ritmo de atendimento. Os dias e horários em que foram colhidos os dados estão representados na tabela 02. 


\begin{tabular}{cc}
\hline \multicolumn{1}{c}{ Dias } & Horário \\
\hline $17 / 11 / 2019$ & $06 \mathrm{~h}$ às $10 \mathrm{~h}$ \\
\hline $18 / 11 / 2019$ & $06 \mathrm{~h}$ às $10 \mathrm{~h}$ \\
\hline $19 / 11 / 2919$ & $06 \mathrm{~h}$ às $10 \mathrm{~h}$ \\
\hline $22 / 11 / 2019$ & $06 \mathrm{~h}$ às $10 \mathrm{~h}$ \\
\hline $25 / 11 / 2019$ & $06 \mathrm{~h}$ às $10 \mathrm{~h}$ \\
\hline
\end{tabular}

Fonte: Autores (2019)

\subsection{Caracterização do Sistema de Filas}

Soncim et al. (2001), destaca que os processos de chegada e atendimento dos usuários do sistema podem ser ajustados através das equações Exponencial Negativa e Poisson, respectivamente, ou através de teste não paramétrico do Qui-Quadrado. Montgomery \& Runger (2002), afirmam que a priori, calculam-se as frequências obtidas para cada intervalo de classe de distribuição desconhecida como $O_{i}$.

Em posse da distribuição hipotética, calculam-se as frequências esperadas aos mesmos intervalos da classe de distribuição desconhecida, determinada como $E_{i}$. A equação 01 então é aplicada.

$$
X_{0}^{2}=\sum_{i=1}^{k} \frac{\left(O_{I}-E_{I}\right)^{2}}{E_{I}}
$$

A hipótese da distribuição desconhecida é aceita como descrita pela distribuição hipotética quando o teste estatístico $X_{0}^{2}<X_{a, k-p-1}^{2}$ é verdadeiro, sendo $a$ o nível de confiança para o teste, $k$ o número de intervalos da classe, $p$ número de parâmetros da distribuição hipotética, valor obtido através da distribuição do Qui- Quadrado para k-p-1 graus de liberdade. A seguir, os gráficos contraídos do software Input Analyzer, com testes para ajuste de curva para procedimento de chegada e atendimento. 


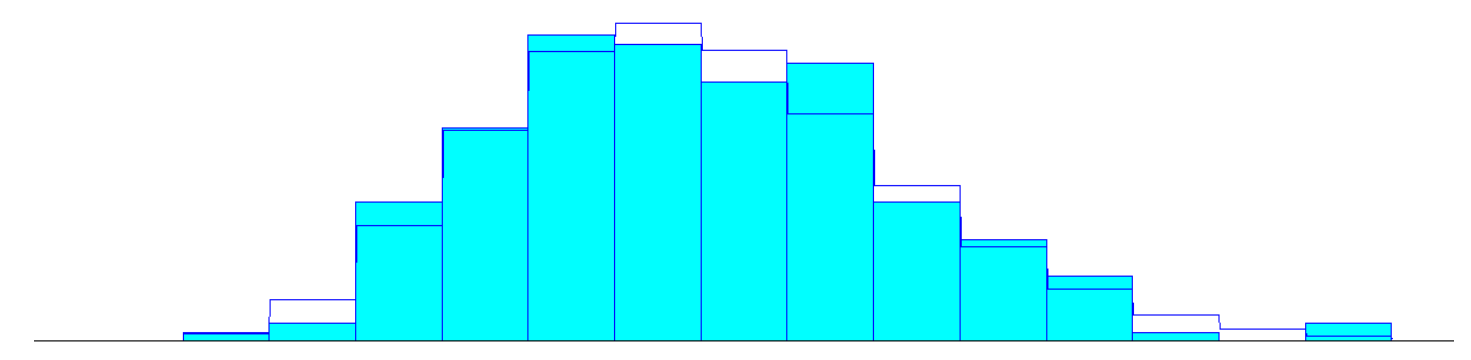

Fonte: Autores (2020)

Tabela 3: Outputs do Input Analyzer (Ritmo de chegada)

\begin{tabular}{|l|l|}
\hline \multicolumn{2}{|c|}{ Sumário da Distribuição } \\
\hline Distribuição: & Poisson \\
\hline Expressão: & POIS(5.49) \\
\hline Erro quadrático: & 0. .po1871 \\
\hline \multicolumn{2}{|c|}{ Test do Chi-Quadrado } \\
\hline Teste Estatístico & $=2.07$ \\
\hline \multicolumn{2}{|c|}{ Sumário dos dados } \\
\hline Número de Pontos de Dados & $=200$ \\
\hline Valor Min nos Dados & $=0$ \\
\hline Valor Max nos Dados & $=13$ \\
\hline Média da Amostra & $=5.49$ \\
\hline Desvio Padrão da Amostra & $=2.32$ \\
\hline & Sumário do Histograma \\
\hline Intervalo do Histograma & $=-0.5$ to 13.5 \\
\hline Número de Intervalos & $=14$ \\
\hline
\end{tabular}

Fonte: Autores (2020)

Figura 04: Gráfico Ritmo de Atendimento

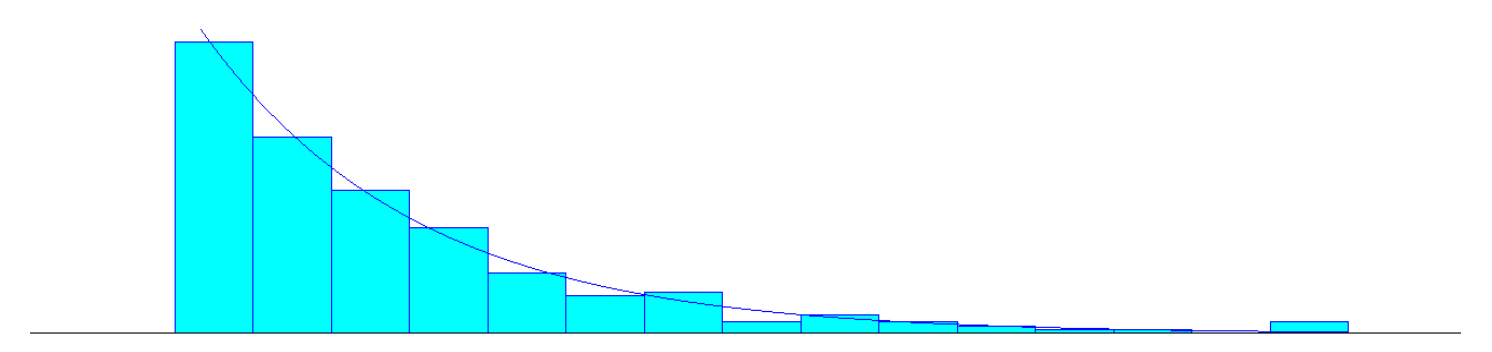

Fonte: Fonte: Autores (2020) 
Tabela 4: Outputs do Input Analyzer (Ritmo de atendimento)

\begin{tabular}{|l|l|}
\hline \multicolumn{2}{|c|}{ Sumário da Distribuição - Gráfico Ritmo de Atendimento } \\
\hline Distribuição: & Exponencial \\
\hline Expressão: & $3+\operatorname{EXPO}(3.86)$ \\
\hline Erro quadrático: & 0.000644 \\
\hline \multicolumn{2}{|c|}{ Test do Chi-Quadrado } \\
\hline Teste Estatístico & $=1.43$ \\
\hline \multicolumn{2}{|c|}{ Sumário do Histograma } \\
\hline Número de Pontos de Dados & $=250$ \\
\hline Valor Min nos Dados & $=3.0$ \\
\hline Valor Max nos Dados & $=23$ \\
\hline
\end{tabular}

Fonte: Adaptado de Input Analyzer - Arena® 11.0 versão Student

O ritmo de atendimento é caracterizado como exponencial, segundo gráfico obtido no Input Analyzer - Arena ${ }^{\circledR}$ 11.0. A partir desses resultados, analisados para o único servidor atendente, a caracterização do sistema é definida pela notação Kendall, da forma

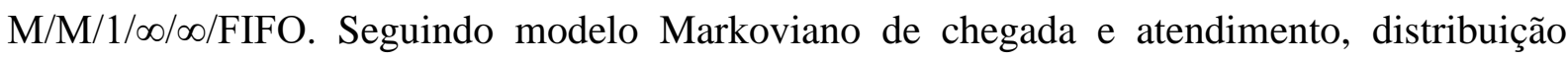
Poisson e Exponencial, respectivamente, com um atendente, considerando a fila e a capacidade do sistema como infinitos, observando que a fila é considerada infinita pois ao sair um usuário um novo entra no sistema e assim sucessivamente, com modelo de fila FIFO, desconsiderando as prioridades, pelo fato de que as fichas prioritárias já são separadas quando os pacientes realizam as fichas no início do atendimento da emergência.

\subsection{Análise do Desempenho Atual}

A partir da coleta dos dados foi possível identificar os ritmos de chegada e atendimento, os dados foram consolidados através do software Excel, assim como aplicação dos ritmos para obtenção dos demais parâmetros de fila. A média entre intervalos de chegada de pessoas para atendimento foi de 5,49 minutos, e do atendimento 3,86 minutos por pessoa. Em posse destes dados, os mesmos foram transformados em ritmo de chegada $(\kappa)$ e ritmo de atendimento $(\mu)$, conforme a tabela 03 abaixo. 
Tabela 03: Medidas do desempenho atual

\begin{tabular}{lcc}
\hline \multicolumn{1}{c}{ Parâmetros } & Servidor & Unidades \\
\hline$K$ & 0,18 & clientes/minutos \\
\hline$\mu$ & 0,26 & clientes $/$ minutos \\
\hline Taxa de utilização do servidor & $69,20 \%$ & $\%$ \\
\hline Número médio de clientes na fila & 1,55 & clientes \\
\hline Probabilidade que o sistema esteja vazio & $30,80 \%$ & minutos \\
\hline Tempo médio de espera na fila & 8,654 & clientes \\
\hline Número médio de clientes no sistema & 2,25 & minutos \\
\hline Tempo médio de espera no sistema & 12,5 & \\
\hline
\end{tabular}

Fonte: Autores (2020)

O número médio de clientes na fila e o tempo médio de espera na fila, analisando de forma subjetiva, não são considerados altos, porém, se tratando de pacientes advindos do pronto socorro, reduções alcançadas nesses tempos, seriam positivas. A média de atendimento do servidor é boa, considerando a média de chegadas de clientes. Ao colher os dados, observouse elevados tempos de atendimento para os pacientes com idade avançadas, mais debilitados ou em bebês e crianças, devido à dificuldade gerada no atendimento desses. O mesmo servidor que realiza a coleta é o que leva o material colhido para o prédio do laboratório, esse deslocamento demanda tempo do servidor, o que pode gerar maior tempo de espera, já que há a possibilidade de chegada de novos usuários para serem atendidos na sua ausência.

Não se limitando a análise dos parâmetros das filas, ainda foram analisados outros aspectos que impactam no tempo de atendimento, como a distribuição dos recursos no ambiente. $\mathrm{O}$ Layout, figura 1, não favorece a otimização do tempo das coletas, pois primeiramente a maca que seria destinada a pacientes com difícil mobilidade como idosos e crianças está sendo utilizada para colocar os materiais de coleta, e a forma como eles são posicionados não beneficia o atendente quando precisa encontrá-los no momento do atendimento fazendo com que haja perda de tempo devido a procura por materiais.

Também foi observado que havia muitas cadeiras na sala de coleta e que a distribuição delas no espaço fazia com que o atendente andasse mais, quando poderiam estar mais próximas dos 
materiais de atendimento. Notou-se ainda que os armários de cabeceira não são a melhor alternativa para guardar os materiais, visto que precisam estar expostos e de fácil visualização, pois quando o atendente precisar possa localizá-los com facilidade.

As lixeiras usadas para descarte de insumos usados poderiam estar alocadas de forma que ficassem mais próximas possível das cadeiras e bancadas, pois evitaria muitos movimentos pelo atendente. Foi observado que não existe um local de espera para os pacientes, geralmente eles aguardam o atendimento no corredor, porém se otimizada a sala de coletas é possível deixar alguns pacientes aguardando na sala agilizando o trabalho do atendente, pois assim que um sair o outro já estará no local para realizar a coleta.

\subsection{Análise de Desempenho Sugerida}

Para otimizar o tempo de atendimento foi elaborada uma proposta de novo Layout, representado na figura 2, com o objetivo de tratar as lacunas observadas no ambiente de atendimento que não contribuem para redução do tempo.

A primeira problemática trabalhada na proposta foi a distribuição dos locais de coleta na sala, apesar de ser um sistema de fila única foi sugerido três locais chamados de "Baias de atendimento", que além de possibilitar a coleta simultânea, casos raros que podem ocorrer em horários de pico onde é adicionado um novo servidor ao sistema, possibilita que pacientes aguardem exatamente no local de atendimento.

Nesse modelo a maca ficaria na Baia 3 , destinada exclusivamente a pacientes que possuem dificuldade de mobilidade, já para o posicionamento dos materiais de coleta sugere-se a substituição dos armários de cabeceira por armários de bancada, pois permitem que os materiais sejam organizados de modo que fiquem visíveis, organizados e de fácil visualização.

Considerou-se também que em cada baia tenha uma cadeira (exceto para a baia 3, visto que no lugar da cadeira seria a maca), uma bancada e uma lixeira, ou seja, o atendente teria todos os recursos próximos. Com essa distribuição seria possível também que algumas cadeiras fossem colocadas na sala para que os próximos pacientes a serem atendidos aguardem no local ao invés de esperarem no corredor, assim visivelmente o servidor teria como observar quantos pacientes aguardam na fila e se precisar de ajuda adicionar um outro ao sistema. Se os pacientes ficarem no corredor junto aos demais não teria essa praticidade de identificar se está 
havendo formação de filas para coleta além do fato que se o paciente tiver dificuldade de se locomover poderia ser atendido no local de espera, ou seja, na sala de coleta.

Figura 05: Proposta novo Layout Sala de Coletas

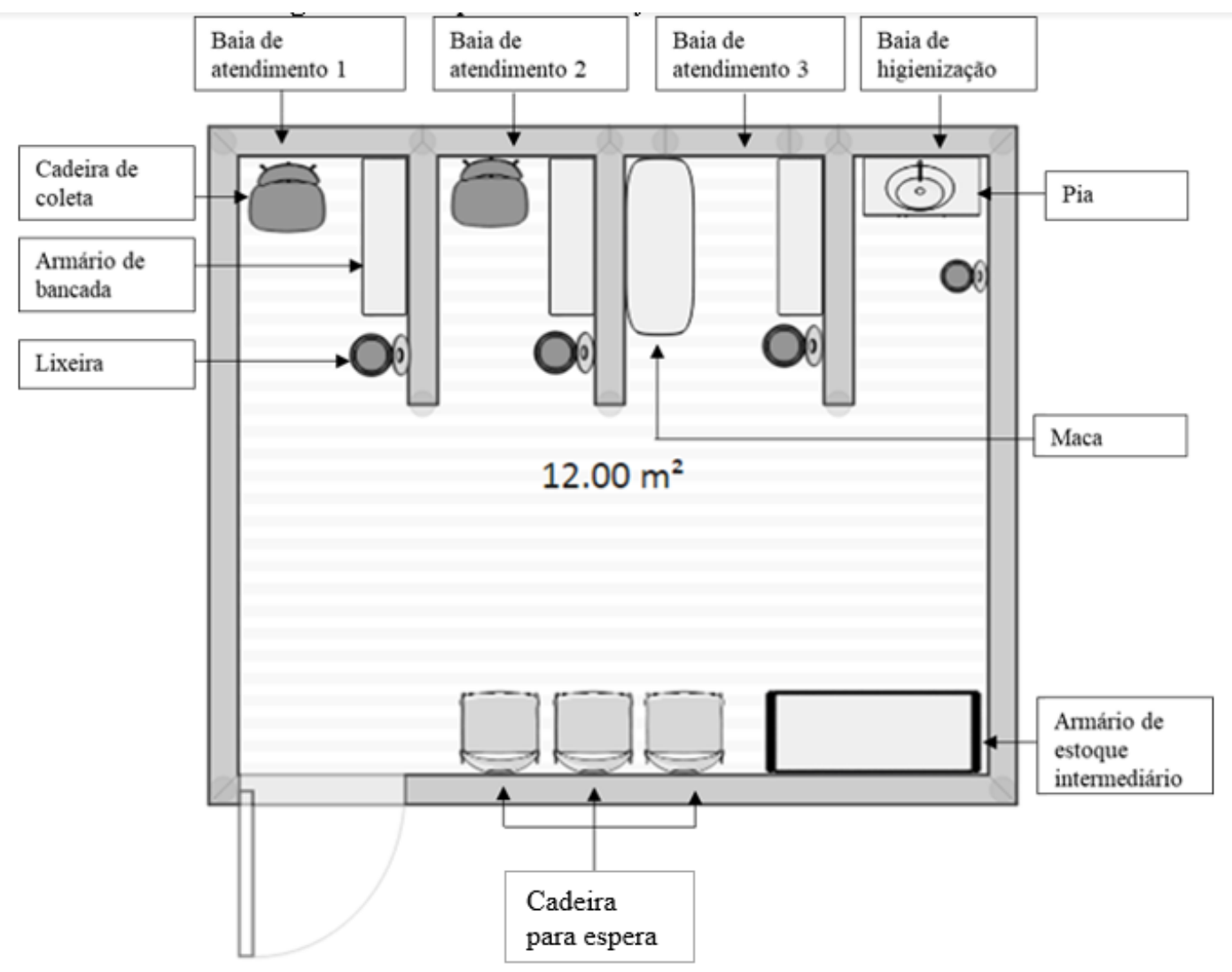

Fonte: Autores, 2019.

\section{Conclusão}

Considerando as análises apresentadas, apesar de o laboratório ser o único do setor público da cidade, foi possível observar que os atendimentos são realizados de forma rápida. Mesmo havendo a ocorrência de filas, o ritmo de atendimento é maior que o ritmo de chegada e o tempo de chegada e o tempo de espera na fila não são considerados longos, porém, podem ser reduzidos.

Por se encontrar nas instalações do hospital municipal, realiza atendimentos e urgência e emergência onde resultados rápidos são de suma importância. Uma forma encontrada é a reorganização do espaço, um novo layout proporcionaria uma redução no tempo de atendimento, otimizando o trabalho dos colaboradores que realizam as coletas, reduzindo consequentemente o tempo de espera dos pacientes.

Ao contrário de muitos setores presentes a rede pública o laboratório municipal consegue suprir sua demanda sem gerar problemas para a população que utiliza seus serviços, mesmo 
com a formação de filas de espera para realização dos exames. Deste modo evidencia-se a necessidade do uso de ferramentas para análise de filas, podendo evidenciar de uma forma eficaz o desempenho com que é realizado. Por ser um setor importante dentro do hospital é necessário um funcionamento perfeito e com agilidade para que pacientes não sejam prejudicados. Assim através de estudos pode ser constatado se o laboratório atende as necessidades dos seus pacientes e garante a qualidade no atendimento por parte dos colaboradores e em caso contrário, ajuda a avaliar melhores alternativas para melhoria e otimização do sistema, podendo ser aplicado nas demais áreas do hospital e em outros setores da rede pública e privada.

\section{REFERÊNCIAS}

ARENALES, M.; ARMENTANO, V.; MORABITO, R. e YANASSE, H. Pesquisa Operacional. Rio de Janeiro: Elsevier, 2007.

CHI, Shih Y.; OLIVEIRA, Bruno A.; PELLISSARI, Joao M. G.; SILVA, Leticia P. e ANDRADE, Fernanda G. Aplicação da Teoria das Filas em um Centro de Atendimento de Saúde em Ponta Grossa - PR. In: XXXIV Encontro Nacional de Engenharia de Produção. Curitiba, 2014.

CAMELO, G. R. COELHO, A, S. BORGES, R. M. SOUZA, R. M. Teoria das filas e da simulação aplicada ao embarque de minério de ferro e manganês no terminal marítimo de ponta da madeira. XXX Encontro Nacional De Engenharia De Produção, São Carlos, 2010. http://www.abepro.org.br/biblioteca/enegep2010_tn_sto_129_830_14824.pdf

DATASUS, Cadastro Nacional de Estabelecimentos de Saúde. Disponível em: <http://cnes2.datasus.gov.br/Listar_Mantidas.asp?VCnpj=22980999000115\&VEstado=15\&VNome=PREFEITU RA\%20MUNICIPAL\%20DE\%20PARAUAPEBAS>. Acesso em 25 de outubro de 2019.

GROSS, D. SHORTLE, J. F. THOMPSON, J. M. HARRIS, C. M. Fundamentals of Queueing Theory. New Jersey: John Wiley \& Sons, 2008.

HILLIER, Frederick S. e LIEBERMAN, Gerald J. Introdução a Pesquisa Operacional. $8^{a}$ Edição. São Paulo: McGraw, 2006.

MARINHO, Alexandre. Um Estudo Sobre as Filas para Internações e para Transplantes no Sistema Único de Saúde Brasileiro. Rio de Janeiro, Instituto de Pesquisa Econômica Aplicada, 2004.

MATHIAS, Rafael V.; COELHO, Antônio S. e GONÇALVES, Mirian B. Desempenho dos Serviços de Internações Hospitalares Utilizando o Modelo de Filas: Estudo de Caso em Hospital de Santa Catarina. In: XII Simpósio de Excelência em Gestão e Tecnologia. Rio de Janeiro, 2015.

PRADO, Darci. Teoria das Filas e Simulação. $2^{\mathrm{a}}$ Edição. Nova Lima, MG: INDG Tecnologia e Serviços LTDA. Série Pesquisa Operacional, Vol.2, 2004.

PLANALTO, Lei N ${ }^{\circ}$ 8.080, De 19 De Setembro De 1990. Disponível em <http://www.planalto.gov.br/ccivil_03/leis/18080.htm>. Acesso em 25 de outubro de 2019.

SONCIM, S. P. ; BRUNS, R. \& SINAY, M. C. F. . Pesquisa operacional: uma aplicação da teoria das filas a um sistema de atendimento. In: XXI Encontro Nacional de Engenharia de Produção. Salvador, 2001. 
TAHA, A. A. Pesquisa Operacional: uma visão geral. 8. ed. São Paulo: Pearson Prentice Hall, 2008 